Bull. Korean Math. Soc. 50 (2013), No. 3, pp. 901-914

http://dx.doi.org/10.4134/BKMS.2013.50.3.901

\title{
INTERPOLATIONS FOR HÖLDER'S INEQUALITY
}

\author{
Kei Ji Izuchi, Kou Hei Izuchi, And Shûichi Ohno
}

Abstract. Kwon and Bae gave an interpolation for a continuous form of Hölder's inequality for a real-valued bounded measurable function on a product of measure spaces. It is given some generalizations of their result.

\section{Introduction}

For real numbers $A$ and $B$ satisfying $A \leq B$, a continuous and increasing function $h$ on the open interval $(0,1)$ is said to be an interpolation for $A \leq B$ if

$$
\lim _{t \rightarrow 0} h(t)=A \quad \text { and } \quad \lim _{t \rightarrow 1} h(t)=B .
$$

Let $(X, \mu)$ be a measure space with $\mu(X)=1$ and $f \in L^{1}(\mu)$ satisfy $f>0$ on $X$. By $[4$, p. 71$]$,

$$
h(t):=\left(\int_{X} f^{t} d \mu\right)^{\frac{1}{t}}, \quad 0<t \leq 1,
$$

is an interpolation for the inequality

$$
\exp \int_{X} \log f d \mu \leq \int_{X} f d \mu
$$

Let $X_{i j}$ and $p_{j}, i=1,2, \ldots, m, j=1,2, \ldots, n$, be positive numbers satisfying

$$
\sum_{j=1}^{n} \frac{1}{p_{j}}=1 \text {. }
$$

The inequality

$$
\sum_{i=1}^{m} \prod_{j=1}^{n} X_{i j} \leq \prod_{j=1}^{n}\left(\sum_{i=1}^{m} X_{i j}^{p_{j}}\right)^{\frac{1}{p_{j}}}
$$

Received April 10, 2012.

2010 Mathematics Subject Classification. Primary 26D15.

Key words and phrases. interpolation, Hölder's inequality, continuous form of Hölder's inequality.

The first author is partially supported by Grant-in-Aid for Scientific Research (No. 21540166), Japan Society for the Promotion of Science. 
is well known as the classical Hölder inequality [1]. In [5], Yang gave an interpolation for Hölder's inequality as follows.

Theorem A. A function

$$
h(t):=\prod_{k=1}^{n}\left[\sum_{i=1}^{m}\left(\prod_{j=1}^{n} X_{i j}\right)^{1-t} X_{i k}^{t p_{k}}\right]^{\frac{1}{p_{k}}}, \quad 0 \leq t \leq 1,
$$

is an interpolation for Hölder's inequality given in (1.1), and $h$ is continuous on $[0,1]$.

Let $(X, \mu)$ and $(Y, \nu)$ be $\sigma$-finite measure spaces with positive measures $\mu, \nu$ and $\mu(X)=1$. Let $(X \times Y, \mu \times \nu)$ be their product measure space. In [2], Kwon gave a continuous form of Hölder's inequality as follows.

Theorem B. Let $\mu(X)=1$ and $f \in L^{1}(\mu \times \nu)$ satisfy $f(x, y)>0$ on $X \times Y$. Then

$$
\begin{aligned}
& \int_{Y} \exp \left(\int_{X} \log f(x, y) d \mu(x)\right) d \nu(y) \\
\leq & \exp \left[\int_{X} \log \left(\int_{Y} f(x, y) d \nu(y)\right) d \mu(x)\right] .
\end{aligned}
$$

Equality holds in (1.2) as a nonzero valued if and only if $f(x, y)=g(x) h(y)$ for $\mu \times \nu$-a.e. on $X \times Y$ with $-\infty<\int_{X} \log g d \mu$.

It may happen that $\log f \notin L^{1}(\mu \times \nu)$; in that case, $\int_{X} \log f(x, y) d \mu(x)$ exists in the extended sense. Since $f \in L^{1}(\mu \times \nu)$,

$$
-\infty \leq \int_{X} \log f(x, y) d \mu(x)<\infty
$$

for $\nu$-a.e. on $Y$. We consider that $\exp (-\infty)=0$ and $\log 0=-\infty$.

Let $\mu(X)=1$ and $f \in L^{1}(\mu \times \nu)$ satisfy $f>0$ on $X \times Y$. Then

$$
-\infty \leq \int_{X} \log f(z, y) d \mu(z) \leq \int_{X} f(z, y) d \mu(z)<\infty .
$$

For $0 \leq t<1$, we define

$$
g(t, x, y):=f^{t}(x, y) \exp \left((1-t) \int_{X} \log f(z, y) d \mu(z)\right)
$$

and

$$
h(t):=\exp \left[\int_{X} \log \left(\int_{Y} g(t, x, y) d \nu(y)\right) d \mu(x)\right] .
$$

When $f=f_{n}$, we write $g_{n}(t, x, y)$ and $h_{n}(t)$. In [3], Kwon and Bae gave an interpolation for the inequality given in (1.2) as follows. 
Theorem C. Let $\mu(X)=1,0<\nu(Y)<\infty$ and $f$ be a measurable function on $X \times Y$ satisfying $0<\delta \leq f(x, y) \leq M<\infty$ for some positive numbers $\delta$ and $M$. In this case, we may define $h$ on $[0,1]$. Then $h$ is an interpolation for the inequality given in (1.2), and $h$ is a continuous and convex function on $[0,1]$.

In this paper, we shall study Theorem $\mathrm{C}$ and relax the assumption " $0<\delta \leq$ $f(x, y) \leq M<\infty "$.

\section{Generalizations of Kwon and Bae's result}

Let $\mu(X)=1$ and $f \in L^{1}(\mu)$ satisfy $f \geq 0$ a.e. on $X$. Then Jensen's inequality [4, Theorem 3.3] says that

$$
\exp \int_{X} \log f d \mu \leq \int_{X} f d \mu .
$$

Lemma 2.1. For $a, b>0$ and $0 \leq t \leq 1$, we have $a^{t} b^{1-t} \leq a+b$.

Proof. We have

$$
a^{t} b^{1-t}=\left(\frac{a}{b}\right)^{t} b \leq \max \{a, b\} \leq a+b .
$$

Lemma 2.2. Let $\mu(X)=1$ and $f \in L^{1}(\mu \times \nu)$ satisfy $f>0$ on $X \times Y$. Then for $0 \leq t<1$, we have the following.

(i) $0 \leq g(t, x, y) \leq f(x, y)+\int_{X} f(z, y) d \mu(z) \in L^{1}(\mu \times \nu)$.

(ii) $\log \int_{Y} g(t, x, y) d \nu(y) \leq \int_{Y} f(x, y) d \nu(y)+\int_{X \times Y} f d \mu \times \nu \in L^{1}(\mu)$.

Proof. (i) By Fubini's theorem, $\int_{X} f(z, y) d \mu(z) \in L^{1}(\nu)$. We have

$$
\begin{aligned}
0 & \leq g(t, x, y)=f^{t}(x, y) \exp \left((1-t) \int_{X} \log f(z, y) d \mu(z)\right) \\
& \leq f^{t}(x, y) \exp \left((1-t) \log \int_{X} f(z, y) d \mu(z)\right) \quad \text { by Jensen's inequality } \\
& =f^{t}(x, y)\left(\int_{X} f(z, y) d \mu(z)\right)^{1-t} \\
& \leq f(x, y)+\int_{X} f(z, y) d \mu(z) \quad \text { by Lemma } 2.1 \\
& \in L^{1}(\mu \times \nu)
\end{aligned}
$$

The second inequality follows from Jensen's inequality.

(ii) By (i), we have

$$
\begin{aligned}
\log \int_{Y} g(t, x, y) d \nu(y) & \leq \int_{Y} g(t, x, y) d \nu(y) \\
& \leq \int_{Y} f(x, y) d \nu(y)+\int_{X \times Y} f d \mu \times \nu \in L^{1}(\mu)
\end{aligned}
$$


Theorem 2.3. Let $\mu(X)=\nu(Y)=1$ and $f \in L^{1}(\mu \times \nu)$ satisfy $\delta \leq f$ on $X \times Y$ for some positive number $\delta$. Then $h$ is an interpolation for the inequality given in (1.2). Moreover $h(1)$ is well defined and $h$ is a continuous and convex function on $[0,1]$.

Proof. We may assume that $0<\delta<1$. For each positive integer $n$, let

$$
f_{n}(x, y):=\min \{f(x, y), n\} .
$$

Then $0<\delta \leq f_{n} \leq n$ and $f_{n} \uparrow f$ as $n \rightarrow \infty$ on $X \times Y$. By Theorem $\mathrm{C}, h_{n}$ is an interpolation for the inequality

$$
\int_{Y} \exp \left(\int_{X} \log f_{n} d \mu\right) d \nu \leq \exp \left[\int_{X} \log \left(\int_{Y} f_{n} d \nu\right) d \mu\right]
$$

and, $h_{n}$ is a continuous and convex function on [0,1]. Letting $n \rightarrow \infty$, by the Lebesgue monotone convergence theorem (we write LMCT in short) we have

$$
\begin{gathered}
\int_{Y} \exp \left(\int_{X} \log f_{n} d \mu\right) d \nu \rightarrow \int_{Y} \exp \left(\int_{X} \log f d \mu\right) d \nu, \\
\exp \left[\int_{X} \log \left(\int_{Y} f_{n} d \nu\right) d \mu\right] \rightarrow \exp \left[\int_{X} \log \left(\int_{Y} f d \nu\right) d \mu\right]
\end{gathered}
$$

and

$$
h_{n}(t) \rightarrow h(t), \quad n \rightarrow \infty
$$

for $0 \leq t \leq 1$. Since $h_{n}$ is a convex and increasing function, $h$ is also a convex and increasing function on $[0,1]$. By $[4$, Theorem 3.2], $h$ is a continuous function on $[0,1)$.

Since $\mu(X)=1$, we have

$$
\begin{aligned}
h(0) & =\exp \left[\int_{X} \log \left(\int_{Y} \exp \left(\int_{X} \log f(z, y) d \mu(z)\right) d \nu(y)\right) d \mu(x)\right] \\
& =\int_{Y} \exp \left(\int_{X} \log f(z, y) d \mu(z)\right) d \nu(y) .
\end{aligned}
$$

Since $\delta \leq f \in L^{1}(\mu \times \nu)$, we have

$$
-\infty<\log \delta \leq \int_{X} \log f(z, y) d \mu(z) \leq \int_{X} f(z, y) d \mu(z)<\infty
$$

for $\nu$-a.e. on $Y$. Hence

$$
h(1)=\exp \left[\int_{X} \log \left(\int_{Y} f(x, y) d \nu(y)\right) d \mu(x)\right]
$$

and

$$
\begin{aligned}
\int_{Y} \exp \left(\int_{X} \log f d \mu\right) d \nu & =h(0) \leq h(t) \leq \lim _{t \rightarrow 1} h(t) \leq h(1) \\
& =\exp \left[\int_{X} \log \left(\int_{Y} f d \nu\right) d \mu\right]
\end{aligned}
$$


To finish the proof, it is sufficient to show that

$$
\lim _{t \rightarrow 1} h(t)=\exp \left[\int_{X} \log \left(\int_{Y} f d \nu\right) d \mu\right] .
$$

Since

$$
0 \leq g(t, x, y)=f^{t}(x, y) \exp \left((1-t) \int_{X} \log f(z, y) d \mu(z)\right) \rightarrow f(x, y)
$$

as $t \rightarrow 1$, by Lemma 2.2(i) and the Lebesgue dominated convergence theorem (we write LDCT in short) we have

$$
\int_{Y} g(t, x, y) d \nu(y) \rightarrow \int_{Y} f(x, y) d \nu(y)
$$

So

$$
\log \int_{Y} g(t, x, y) d \nu(y) \rightarrow \log \int_{Y} f(x, y) d \nu(y)
$$

as $t \rightarrow 1$ for $\mu$-a.e. on $X$. Since $\delta=\delta^{t} \delta^{1-t} \leq g(t, x, y)$,

$$
-\infty<\log \delta \leq \log \int_{Y} g(t, x, y) \nu(y) .
$$

Hence by Lemma 2.2(ii) and LDCT, we get (2.1).

Theorem 2.4. Let $\mu(X)=\nu(Y)=1$ and $f \in L^{1}(\mu \times \nu)$ satisfy $f>0$ on $X \times Y$. Then $h$ is a continuous, convex and increasing function on $[0,1)$, and $\int_{Y} \exp \left(\int_{X} \log f d \mu\right) d \nu=h(0) \leq h(t) \leq \lim _{t \rightarrow 1} h(t) \leq \exp \left[\int_{X} \log \left(\int_{Y} f d \nu\right) d \mu\right]$.

Proof. For each positive integer $N$, let

$$
f_{N}(x, y)=\max \left\{f(x, y), \frac{1}{N}\right\} .
$$

Then $0<1 / N \leq f_{N} \in L^{1}(\mu \times \nu)$ and $f_{N} \downarrow f$ as $N \rightarrow \infty$ on $X \times Y$. By Theorem $2.3, h_{N}$ is an interpolation for the inequality

$$
\int_{Y} \exp \left(\int_{X} \log f_{N} d \mu\right) d \nu \leq \exp \left[\int_{X} \log \left(\int_{Y} f_{N} d \nu\right) d \mu\right],
$$

and $h_{N}$ is a continuous and convex function on [0,1]. Since $\log f_{N} \downarrow \log f$ and $\log f_{N} \leq \log f_{1} \leq f_{1} \in L^{1}(\mu \times \nu)$, by LMCT we have

$$
\exp \left(\int_{X} \log f_{N} d \mu\right) \downarrow \exp \left(\int_{X} \log f d \mu\right) \text {. }
$$

By Jensen's inequality,

$$
\begin{aligned}
\exp \left(\int_{X} \log f_{N}(x, y) d \mu(x)\right) & \leq \int_{X} f_{N}(x, y) d \mu(x) \\
& \leq \int_{X} f_{1}(x, y) d \mu(x) \in L^{1}(\nu)
\end{aligned}
$$


Hence by LMCT, we have

$$
\int_{Y} \exp \left(\int_{X} \log f_{N} d \mu\right) d \nu \rightarrow \int_{Y} \exp \left(\int_{X} \log f d \mu\right) d \nu .
$$

Since

$$
\log \left(\int_{Y} f_{N} d \nu\right) \downarrow \log \left(\int_{Y} f d \nu\right)
$$

and

$$
\log \int_{Y} f_{N} d \nu \leq \int_{Y} f_{N} d \nu \leq \int_{Y} f_{1} d \nu \in L^{1}(\mu)
$$

by LMCT we have

$$
\int_{X} \log \left(\int_{Y} f_{N} d \nu\right) d \mu \rightarrow \int_{X} \log \left(\int_{Y} f d \nu\right) d \mu
$$

so

$$
h_{N}(1)=\exp \left[\int_{X} \log \left(\int_{Y} f_{N} d \nu\right) d \mu\right] \rightarrow \exp \left[\int_{X} \log \left(\int_{Y} f d \nu\right) d \mu\right] .
$$

Let fix $t$ with $0 \leq t<1$. By (1.3), we have

$$
0<g_{N}(t, x, y) \downarrow g(t, x, y), \quad N \rightarrow \infty .
$$

By Lemma 2.2(i) and LMCT, we have

$$
\log \int_{Y} g_{N}(t, x, y) d \nu(y) \downarrow \log \int_{Y} g(t, x, y) d \nu(y) .
$$

By Lemma 2.2(ii),

$$
\begin{aligned}
\log \int_{Y} g_{N}(t, x, y) d \nu(y) & \leq \int_{Y} f_{N}(x, y) d \nu(y)+\int_{X \times Y} f_{N} d \mu \times \nu \\
& \leq \int_{Y} f_{1}(x, y) d \nu(y)+\int_{X \times Y} f_{1} d \mu \times \nu \\
& \in L^{1}(\mu) .
\end{aligned}
$$

By (1.4) and LMCT, we have $h_{N}(t) \rightarrow h(t)$ as $N \rightarrow \infty$ for $0 \leq t<1$. Then $h$ is a convex and increasing function on $[0,1)$, so $h$ is continuous on $[0,1)$. We note that

$$
h(0)=\int_{Y} \exp \left(\int_{X} \log f d \mu\right) d \nu
$$

Since

we have

$$
h_{N}(t) \leq h_{N}(1)=\exp \left[\int_{X} \log \left(\int_{Y} f_{N} d \nu\right) d \mu\right]
$$

$$
h(t) \leq \exp \left[\int_{X} \log \left(\int_{Y} f d \nu\right) d \mu\right], \quad 0 \leq t<1 .
$$

Therefore we get our assertion. 
Suppose that $0<\nu(Y)<\infty$. We have

$$
\begin{aligned}
\int_{Y} \exp \left(\int_{X} \log f d \mu\right) \frac{d \nu}{\nu(Y)} & =\frac{1}{\nu(Y)} \int_{Y} \exp \left(\int_{X} \log f d \mu\right) d \nu, \\
\exp \left[\int_{X} \log \left(\int_{Y} f \frac{d \nu}{\nu(Y)}\right) d \mu\right] & =\frac{1}{\nu(Y)} \exp \left[\int_{X} \log \left(\int_{Y} f d \nu\right) d \mu\right]
\end{aligned}
$$

and

$$
\begin{aligned}
& \exp \left[\int_{X} \log \left(\int_{Y} g(t, x, y) \frac{d \nu(y)}{\nu(Y)}\right) d \mu(x)\right] \\
= & \frac{1}{\nu(Y)} \exp \left[\int_{X} \log \left(\int_{Y} g(t, x, y) d \nu(y)\right) d \mu(x)\right] .
\end{aligned}
$$

By Theorem 2.4, we have the following.

Corollary 2.5. Let $\mu(X)=1,0<\nu(Y)<\infty$ and $f \in L^{1}(\mu \times \nu)$ satisfy $f>0$ on $X \times Y$. Then $h$ is a continuous, convex and increasing function on $[0,1)$, and

$\int_{Y} \exp \left(\int_{X} \log f d \mu\right) d \nu=h(0) \leq h(t) \leq \lim _{t \rightarrow 1} h(t) \leq \exp \left[\int_{X} \log \left(\int_{Y} f d \nu\right) d \mu\right]$.

Next, we shall study the case of $\nu(Y)=\infty$.

Theorem 2.6. Let $\mu(X)=1,0<\nu(Y) \leq \infty$ and $f \in L^{1}(\mu \times \nu)$ satisfy $f>0$ on $X \times Y$. Then $h$ is a continuous, convex and increasing function on $[0,1)$, and

$\int_{Y} \exp \left(\int_{X} \log f d \mu\right) d \nu=h(0) \leq h(t) \leq \lim _{t \rightarrow 1} h(t) \leq \exp \left[\int_{X} \log \left(\int_{Y} f d \nu\right) d \mu\right]$.

Proof. By Corollary 2.5, it is sufficient to show the case of $\nu(Y)=\infty$.

First, suppose that

$$
\int_{Y} \exp \left(\int_{X} \log f d \mu\right) d \nu=0
$$

Hence

$$
\int_{X} \log f(z, y) d \mu(z)=-\infty
$$

$\nu$-a.e. on $Y$. So by (1.3), $g(t, x, y)=0$ for $\mu \times \nu$-a.e. on $X \times Y$ and $0 \leq t<1$.

Thus by (1.4), we get $h(t)=0$ for $0 \leq t<1$ and the assertion.

Next, suppose that

$$
0<\int_{Y} \exp \left(\int_{X} \log f d \mu\right) d \nu
$$

By Jensen's inequality,

$$
0<\int_{Y} \exp \left(\int_{X} \log f d \mu\right) d \nu \leq \int_{X \times Y} f d \mu \times \nu<\infty .
$$


Since $\nu$ is a $\sigma$-finite measure, there is a sequence of measurable subsets $\left\{Y_{n}\right\}_{n}$ of $Y$ such that $Y_{n} \subset Y_{n+1}, \nu\left(Y_{n}\right)<\infty$ for every $n \geq 1, Y=\bigcup_{n=1}^{\infty} Y_{n}$ and by $(2.2)$,

$$
0<\int_{Y_{1}} \exp \left(\int_{X} \log f d \mu\right) d \nu
$$

Let

$$
h_{n}(t):=\exp \left[\int_{X} \log \left(\int_{Y_{n}} g(t, x, y) d \nu(y)\right) d \mu(x)\right], \quad 0 \leq t<1 .
$$

By Corollary 2.5, $h_{n}$ is a continuous, convex and increasing function on $[0,1)$, and

$$
\begin{aligned}
\int_{Y_{n}} \exp \left(\int_{X} \log f d \mu\right) d \nu & =h_{n}(0) \leq h_{n}(t) \leq \lim _{t \rightarrow 1} h_{n}(t) \\
& \leq \exp \left[\int_{X} \log \left(\int_{Y_{n}} f d \nu\right) d \mu\right]<\infty .
\end{aligned}
$$

For each $y \in Y$, we have

$$
0 \leq \chi_{Y_{n}}(y) \exp \left(\int_{X} \log f(z, y) d \mu(z)\right) \uparrow \exp \left(\int_{X} \log f(z, y) d \mu(z)\right) .
$$

By LMCT,

$$
\int_{Y_{n}} \exp \left(\int_{X} \log f d \mu\right) d \nu \uparrow \int_{Y} \exp \left(\int_{X} \log f d \mu\right) d \nu .
$$

By (2.3) and (2.4),

$$
\log \int_{Y_{1}} f d \nu \in L^{1}(\mu)
$$

We have

$$
\log \int_{Y_{1}} f d \nu \leq \log \int_{Y_{n}} f d \nu \uparrow \log \int_{Y} f d \nu
$$

for $\mu$-a.e. on $X$. By LMCT, we have

$$
\exp \left[\int_{X} \log \left(\int_{Y_{n}} f d \nu\right) d \mu\right] \uparrow \exp \left[\int_{X} \log \left(\int_{Y} f d \nu\right) d \mu\right] .
$$

In the similar way, we obtain

$$
h_{n}(t) \uparrow h(t), \quad 0 \leq t<1 .
$$

By $(2.4), h$ is a continuous, convex and increasing function on $[0,1)$, and

$$
\begin{aligned}
\int_{Y} \exp \left(\int_{X} \log f d \mu\right) d \nu & =h(0) \leq h(t) \leq \lim _{t \rightarrow 1} h(t) \\
& \leq \exp \left[\int_{X} \log \left(\int_{Y} f d \nu\right) d \mu\right] .
\end{aligned}
$$




\section{Interpolations for Hölder's inequality}

Let $\mu(X)=1,0<\nu(Y) \leq \infty$ and $f \in L^{1}(\mu \times \nu)$ satisfy $f>0$ on $X \times Y$. If

$$
\exp \left[\int_{X} \log \left(\int_{Y} f d \nu\right) d \mu\right]=0,
$$

then by Theorem 2.6, trivially $h$ is an interpolation for (1.2). Since

$$
\log \int_{Y} f d \nu \leq \int_{Y} f d \nu \in L^{1}(\mu)
$$

if $\log \int_{Y} f d \nu \notin L^{1}(\mu)$, then

$$
\exp \left[\int_{X} \log \left(\int_{Y} f d \nu\right) d \mu\right]=0,
$$

so we assume that

$$
\log \int_{Y} f d \nu \in L^{1}(\mu)
$$

Let

$$
Y_{\infty}:=\left\{y \in Y: \int_{X} \log f(z, y) d \mu(z) \neq-\infty\right\} .
$$

Suppose that $0<\nu\left(Y \backslash Y_{\infty}\right)$. Then for $0 \leq t<1$, we have

$$
\begin{gathered}
\int_{Y} \exp \left(\int_{X} \log f d \mu\right) d \nu=\int_{Y_{\infty}} \exp \left(\int_{X} \log f d \mu\right) d \nu \\
\int_{Y} f^{t} \exp \left((1-t) \int_{X} \log f d \mu\right) d \nu=\int_{Y_{\infty}} f^{t} \exp \left((1-t) \int_{X} \log f d \mu\right) d \nu \\
h(t)=\exp \left[\int_{X} \log \left(\int_{Y_{\infty}} g(t, x, y) d \nu\right) d \mu\right]
\end{gathered}
$$

and by (3.1),

$$
\exp \left[\int_{X} \log \left(\int_{Y_{\infty}} f d \nu\right) d \mu\right]<\exp \left[\int_{X} \log \left(\int_{Y} f d \nu\right) d \mu\right] .
$$

By Theorem 2.6, we have the following.

Corollary 3.1. Let $\mu(X)=1,0<\nu(Y) \leq \infty$ and $f \in L^{1}(\mu \times \nu)$ satisfy $f>0$ on $X \times Y$. If (3.1) holds and $0<\nu\left(Y \backslash Y_{\infty}\right)$, then

$$
\begin{aligned}
& \int_{Y} \exp \left(\int_{X} \log f d \mu\right) d \nu=h(0) \leq h(t) \leq \lim _{t \rightarrow 1} h(t) \\
\leq & \exp \left[\int_{X} \log \left(\int_{Y_{\infty}} f d \nu\right) d \mu\right]<\exp \left[\int_{X} \log \left(\int_{Y} f d \nu\right) d \mu\right] .
\end{aligned}
$$

So $h$ is not an interpolation for the inequality given in (1.2).

Hence for the study of interpolations, we may assume that

$$
\nu\left(Y \backslash Y_{\infty}\right)=0 \text {. }
$$


Lemma 3.2. Let $\mu(X)=1,0<\nu(Y) \leq \infty$ and $f \in L^{1}(\mu \times \nu)$ satisfy $f>0$ on $X \times Y$. If (3.2) holds and there is a function $F(x) \in L^{1}(\mu)$ such that

$$
F(x) \leq \log \int_{Y} g(t, x, y) d \nu(y)
$$

for every $0 \leq t<1$, then $h$ is an interpolation for Hölder's inequality given in $(1.2)$, and $h$ is a convex function on $[0,1)$.

Proof. By Theorem 2.6, it is sufficient to show that

$$
\lim _{t \rightarrow 1} h(t)=\exp \left[\int_{X} \log \left(\int_{Y} f d \nu\right) d \mu\right] .
$$

By $(3.2)$,

$$
-\infty<\int_{X} \log f(z, y) d \mu(z)<\infty
$$

for $\nu$-a.e. on $Y$. Hence by (1.3),

$$
0 \leq g(t, x, y) \rightarrow f(x, y), \quad t \rightarrow 1
$$

for $\mu \times \nu$-a.e. on $X \times Y$. By Lemma 2.2(i) and LDCT, we have

$$
\log \int_{Y} g(t, x, y) d \nu(y) \rightarrow \log \int_{Y} f(x, y) d \nu(y), \quad t \rightarrow 1 .
$$

By Lemma 2.2(ii), the assumption and LDCT, we get

$$
\begin{aligned}
h(t) & =\exp \left[\int_{X} \log \left(\int_{Y} g(t, x, y) d \nu(y)\right) d \mu(x)\right] \\
& \rightarrow \exp \left[\int_{X} \log \left(\int_{Y} f d \nu\right) d \mu\right], \quad t \rightarrow 1 .
\end{aligned}
$$

Theorem 3.3. Let $\mu(X)=1$ and $0<\nu(Y) \leq \infty$. Let $\varphi_{1}, \varphi_{2}, \ldots, \varphi_{n}$ be real valued functions in $L^{1}(\mu)$ and $\psi_{1}, \psi_{2}, \ldots, \psi_{n}$ be real valued measurable functions on $Y$. Let

$$
f(x, y)=\exp \sum_{j=1}^{n} \varphi_{j}(x) \psi_{j}(y) .
$$

Suppose that $f \in L^{1}(\mu \times \nu)$. Then $h$ is an interpolation for Hölder's inequality given in $(1.2)$, and $h$ is a convex function on $[0,1)$.

Proof. We have

$$
\int_{X} \log f(z, y) d \mu(z)=\sum_{j=1}^{n} \psi_{j}(y) \int_{X} \varphi_{j}(z) d \mu(z) .
$$

Hence (3.2) holds and by (1.3),

$$
g(t, x, y)=\exp \left(t \sum_{j=1}^{n} \varphi_{j}(x) \psi_{j}(y)+(1-t) \sum_{j=1}^{n} \psi_{j}(y) \int_{X} \varphi_{j} d \mu\right)
$$




$$
\begin{aligned}
& =\exp \left(\sum_{j=1}^{n} \psi_{j}(y)\left(t \varphi_{j}(x)+(1-t) \int_{X} \varphi_{j} d \mu\right)\right) \\
& \geq \exp \left(-\sum_{j=1}^{n}\left|\psi_{j}(y)\right|\left(\left|\varphi_{j}(x)\right|+\left|\int_{X} \varphi_{j} d \mu\right|\right)\right) .
\end{aligned}
$$

Hence for $0<\lambda<\infty$, we have

$$
\begin{aligned}
& \int_{Y} g(t, x, y) d \nu(y) \\
\geq & \int_{Y} \exp \left(-\sum_{j=1}^{n}\left|\psi_{j}(y)\right|\left(\left|\varphi_{j}(x)\right|+\left|\int_{X} \varphi_{j} d \mu\right|\right)\right) d \nu(y) \\
\geq & \int_{\bigcap_{j=1}^{n}\left\{\left|\psi_{j}\right| \leq \lambda\right\}} \exp \left(-\sum_{j=1}^{n}\left|\psi_{j}(y)\right|\left(\left|\varphi_{j}(x)\right|+\left|\int_{X} \varphi_{j} d \mu\right|\right)\right) d \nu(y) \\
\geq & \nu\left(\bigcap_{j=1}^{n}\left\{\left|\psi_{j}\right| \leq \lambda\right\}\right) \times \exp \left(-\lambda \sum_{j=1}^{n}\left(\left|\varphi_{j}(x)\right|+\left|\int_{X} \varphi_{j} d \mu\right|\right)\right) .
\end{aligned}
$$

Since $f \in L^{1}(\mu \times \nu)$ and $\varphi_{j} \in L^{1}(\mu)$ for $1 \leq j \leq n$, by Lemma 2.2(ii) we have

$$
\nu\left(\bigcap_{j=1}^{n}\left\{\left|\psi_{j}\right| \leq \lambda\right\}\right)<\infty .
$$

Since $\psi_{1}, \psi_{2}, \ldots, \psi_{n}$ are real valued measurable functions on $Y$,

$$
0<\nu\left(\bigcap_{j=1}^{n}\left\{\left|\psi_{j}\right| \leq \lambda\right\}\right)
$$

for some $0<\lambda<\infty$. Therefore

$$
\begin{aligned}
& \log \int_{Y} g(t, x, y) d \nu(y) \\
\geq & \log \nu\left(\bigcap_{j=1}^{n}\left\{\left|\psi_{j}\right| \leq \lambda\right\}\right)-\lambda \sum_{j=1}^{n}\left(\left|\varphi_{j}(x)\right|+\left|\int_{X} \varphi_{j} d \mu\right|\right) \\
\in & L^{1}(\mu) \quad \text { by the assumption. }
\end{aligned}
$$

By Lemma 3.2, we get the assertion.

Corollary 3.4. Let $\mu(X)=1,0<\nu(Y) \leq \infty$ and $f \in L^{1}(\mu \times \nu)$ satisfy $f>0$ on $X \times Y$. Suppose that there are real valued functions $\varphi_{1}, \varphi_{2}, \ldots, \varphi_{n} \in L^{1}(\mu)$ and real valued measurable functions $\psi_{1}, \psi_{2}, \ldots, \psi_{n}$ on $Y$ such that

$$
\sum_{j=1}^{n} \varphi_{j}(x) \psi_{j}(y) \leq \log f(x, y) .
$$

Then $h$ is an interpolation for Hölder's inequality given in (1.2), and $h$ is a convex function on $[0,1)$. 
Proof. We have

$$
\exp \sum_{j=1}^{n} \varphi_{j}(x) \psi_{j}(y) \leq f(x, y)
$$

and

$$
\sum_{j=1}^{n} \psi_{j}(y) \int_{X} \varphi_{j}(z) d \mu(z) \leq \int_{X} \log f(z, y) d \mu(z)
$$

Hence

$$
\begin{aligned}
& \log \int_{Y} \exp \left(\sum_{j=1}^{n} \psi_{j}(y)\left(t \varphi_{j}(x)+(1-t) \int_{X} \varphi_{j} d \mu\right)\right) d \nu(y) \\
\leq & \log \int_{Y} g(t, x, y) d \nu(y) .
\end{aligned}
$$

By the proof of Theorem 3.3, there exists $F(x) \in L^{1}(\mu)$ satisfying

$$
F(x) \leq \log \int_{Y} \exp \left(\sum_{j=1}^{n} \psi_{j}(y)\left(t \varphi_{j}(x)+(1-t) \int_{X} \varphi_{j} d \mu\right)\right) d \nu(y)
$$

for every $0 \leq t<1$. By Lemma 3.2, we get the assertion.

Example 3.5. Let $X=\{1,2\}$ and $0<\lambda<1$. Let $\mu$ be the measure on $X$ satisfying $\mu(\{1\})=\lambda$ and $\mu(\{2\})=1-\lambda$. Let $\nu$ be a $\sigma$-finite positive measure on $Y$. Let

$$
f(x, y)=e^{\chi_{\{1\}} \psi_{1}(y)+\chi_{\{2\}} \psi_{2}(y)}
$$

for real valued measurable functions $\psi_{1}, \psi_{2}$ on $Y$. Then we have

$$
\int_{Y} \exp \left(\int_{X} \log f d \mu\right) d \nu=\int_{Y}\left(e^{\psi_{1}}\right)^{\lambda}\left(e^{\psi_{2}}\right)^{1-\lambda} d \nu
$$

and

$$
\exp \left[\int_{X} \log \left(\int_{Y} f d \nu\right) d \mu\right]=\left(\int_{Y} e^{\psi_{1}} d \nu\right)^{\lambda}\left(\int_{Y} e^{\psi_{2}} d \nu\right)^{1-\lambda} .
$$

We have also that $f \in L^{1}(\mu \times \nu)$ if and only if $e^{\psi_{1}}, e^{\psi_{2}} \in L^{1}(\nu)$. Under the condition that $e^{\psi_{1}}, e^{\psi_{2}} \in L^{1}(\nu)$, for $0 \leq t<1$ by Theorem 3.3

$$
h(t)=\left(\int_{Y} e^{t \psi_{1}+(1-t)\left(\lambda \psi_{1}+(1-\lambda) \psi_{2}\right)} d \nu\right)^{\lambda}\left(\int_{Y} e^{t \psi_{2}+(1-t)\left(\lambda \psi_{1}+(1-\lambda) \psi_{2}\right)} d \nu\right)^{1-\lambda}
$$

is an interpolation for Hölder's inequality;

$$
\int_{Y}\left(e^{\psi_{1}}\right)^{\lambda}\left(e^{\psi_{2}}\right)^{1-\lambda} d \nu \leq\left(\int_{Y} e^{\psi_{1}} d \nu\right)^{\lambda}\left(\int_{Y} e^{\psi_{2}} d \nu\right)^{1-\lambda}
$$

In the case of $0<\nu(Y)<\infty$, we have another condition on $f$ for the interpolation.

Proposition 3.6. Let $\mu(X)=1,0<\nu(Y)<\infty$ and $f \in L^{1}(\mu \times \nu)$ satisfy $f>0$ on $X \times Y$ and $\log f \in L^{1}(\mu \times \nu)$. Then $h$ is an interpolation for the inequality given in (1.2), and $h$ is a convex function on $[0,1)$. 
Proof. By Fubini's theorem,

$$
\int_{X} \log f(z, y) d \mu(z) \in L^{1}(\nu)
$$

so (3.2) holds. By Jensen's inequality, we have

$$
\begin{aligned}
& \log \int_{Y} g(t, x, y) \frac{d \nu(y)}{\nu(Y)} \\
\geq & \int_{Y} \log g(t, x, y) \frac{d \nu(y)}{\nu(Y)} \\
= & \int_{Y}\left(t \log f(x, y)+(1-t) \int_{X} \log f(z, y) d \mu(z)\right) \frac{d \nu(y)}{\nu(Y)} \\
= & t \int_{Y} \log f(x, y) \frac{d \nu(y)}{\nu(Y)}+(1-t) \int_{X \times Y} \log f \frac{d \mu \times \nu}{\nu(Y)} \\
\geq & -\int_{Y}|\log f(x, y)| \frac{d \nu(y)}{\nu(Y)}-\int_{X \times Y}|\log f| \frac{d \mu \times \nu}{\nu(Y)} \\
& \in L^{1}(\mu) .
\end{aligned}
$$

By Lemma 3.2, we get the assertion.

In the case of $\nu(Y)=\infty$, Proposition 3.6 does not have meaning. For, there are no functions $f \in L^{1}(\mu \times \nu)$ satisfying $f>0$ on $X \times Y$ and $\log f \in L^{1}(\mu \times \nu)$. If $f \in L^{1}(\mu \times \nu)$, then

$$
(\mu \times \nu)(\{f \geq 1 / 2\})<\infty
$$

SO

$$
(\mu \times \nu)(\{f<1 / 2\})=\infty
$$

Hence

$$
-\int_{\{f<1 / 2\}} \log f d \mu \times \nu>(\log 2)(\mu \times \nu)(\{f<1 / 2\})=\infty .
$$

Therefore $\log f \notin L^{1}(\mu \times \nu)$.

\section{References}

[1] G. H. Hardy, J. E. Littlewood, and G. Pólya, Inequalities, Cambridge University Press, Cambridge, 1952.

[2] E. G. Kwon, Extension of Hölder's inequality. I, Bull. Austral. Math. Soc. 51 (1995), 369-375.

[3] E. G. Kwon and E. K. Bae, On a continuous form of Hölder inequality, J. Math. Anal. Appl. 343 (2008), no. 1, 585-592.

[4] W. Rudin, Real and Complex Analysis, Third Edition, McGraw-Hill, New York, 1987.

[5] X. Yang, A generalization of Hölder's inequality, J. Math. Anal. Appl. 247 (2000), no. $1,328-330$. 
KEI Ji IZUCHI

Department of Mathematics

NiIgATA University

NiIGATA 950-2181, JAPAN

E-mail address: izuchi@m.sc.niigata-u.ac.jp

Kou Hei Izuchi

Department of Mathematics

FACULTY OF EDUCATION

YAMAGUCHI UNIVERSITY

YAMAGUCHI 753-8511, JAPAN

E-mail address: izuchi@yamaguchi-u.ac.jp

SHÛICHI OHNO

Nippon Institute of Technology

Miyashiro, Minami-Saitama 345-8501, JAPAN

E-mail address: ohno@nit.ac.jp 